University of Warwick institutional repository: http://go.warwick.ac.uk/wrap This paper is made available online in accordance with publisher policies. Please scroll down to view the document itself. Please refer to the repository record for this item and our policy information available from the repository home page for further information.

To see the final version of this paper please visit the publisher's website. Access to the published version may require a subscription.

Author(s): M. CSÖRNYEI, T. JORDAN, M. POLLICOTT, D. PREISS and B. SOLOMYAK

Article Title: Positive-measure self-similar sets without interior

Year of publication: 2006

Link to published

version: http://dx.doi.org/10.1017/S0143385705000702

Publisher statement: None 


\title{
Positive-measure self-similar sets without interior
}

\author{
M. CSÖRNYEI $\dagger$, T. JORDAN $\ddagger$, M. POLLICOTT $\ddagger$ D. PREISS $\dagger$ and B. SOLOMYAK $\S$ \\ $\dagger$ Department of Mathematics, University College London, Gower Street, \\ London WC1E 6BT, UK \\ (e-mail: $\{$ m.csornyei,dp\}@ucl.ac.uk) \\ $\ddagger$ Department of Mathematics, Warwick University, Coventry CV4 7AL, UK \\ (e-mail: \{tjordan, mpollic\}@maths.warwick.ac.uk) \\ $\S$ Department of Mathematics, University of Washington, Box 354350, Seattle, \\ WA 98195-4350, USA \\ (e-mail: solomyak@math.washington.edu)
}

(Received 23 June 2004 and accepted in revised form 29 November 2004)

\begin{abstract}
We recall the problem posed by Peres and Solomyak in Problems on self-similar and self-affine sets; an update. Progr. Prob. 46 (2000), 95-106: can one find examples of self-similar sets with positive Lebesgue measure, but with no interior? The method in Properties of measures supported on fat Sierpinski carpets, this issue, leads to families of examples of such sets.

In [1] the existence of self-affine sets with positive Lebesgue measure but empty interior was shown. In this appendix we demonstrate that an adjustment of this argument can show the existence of self-similar sets with positive Lebesgue measure but empty interior answering a question from [2].
\end{abstract}

The construction. Let $\underline{t}=\left(t_{1}, t_{2}\right) \in[0,1]^{2} \subset \mathbb{R}^{2}$. We consider ten similarities (with the same contraction rate $\frac{1}{3}$ ) given by

$$
\begin{array}{ll}
T_{0}(x, y)=\left(\frac{1}{3} x, \frac{1}{3} y\right), & T_{5}(x, y)=\left(\frac{1}{3}+\frac{1}{3} x, \frac{1}{3} y+1\right) \\
T_{1}(x, y)=\left(\frac{1}{3} x, \frac{1}{3} y+t_{1}\right), & T_{6}(x, y)=\left(\frac{2}{3}+\frac{1}{3} x, \frac{1}{3} y\right) \\
T_{2}(x, y)=\left(\frac{1}{3} x, \frac{1}{3} y+t_{2}\right), & T_{7}(x, y)=\left(\frac{2}{3}+\frac{1}{3} x, \frac{1}{3} y+t_{1}\right) \\
T_{3}(x, y)=\left(\frac{1}{3} x, \frac{1}{3} y+1\right), & T_{8}(x, y)=\left(\frac{2}{3}+\frac{1}{3} x, \frac{1}{3} y+t_{2}\right) \\
T_{4}(x, y)=\left(\frac{1}{3}+\frac{1}{3} x, \frac{1}{3} y\right), & T_{9}(x, y)=\left(\frac{2}{3}+\frac{1}{3} x, \frac{1}{3} y+1\right) .
\end{array}
$$

This construction is similar in spirit to those in [3]. To see that the associated limit set $\Lambda_{\underline{t}}$ has empty interior, observe that the intersection of $\Lambda_{\underline{t}}$ with each of vertical lines $\left\{\left(\bar{k}+\frac{1}{2}\right) 3^{-n}\right\} \times \mathbb{R}$, with $n \geq 0$ and $0 \leq k \leq 3^{n}-1$ has zero measure. It remains to show that typically $\Lambda_{\underline{t}}$ has positive measure. 


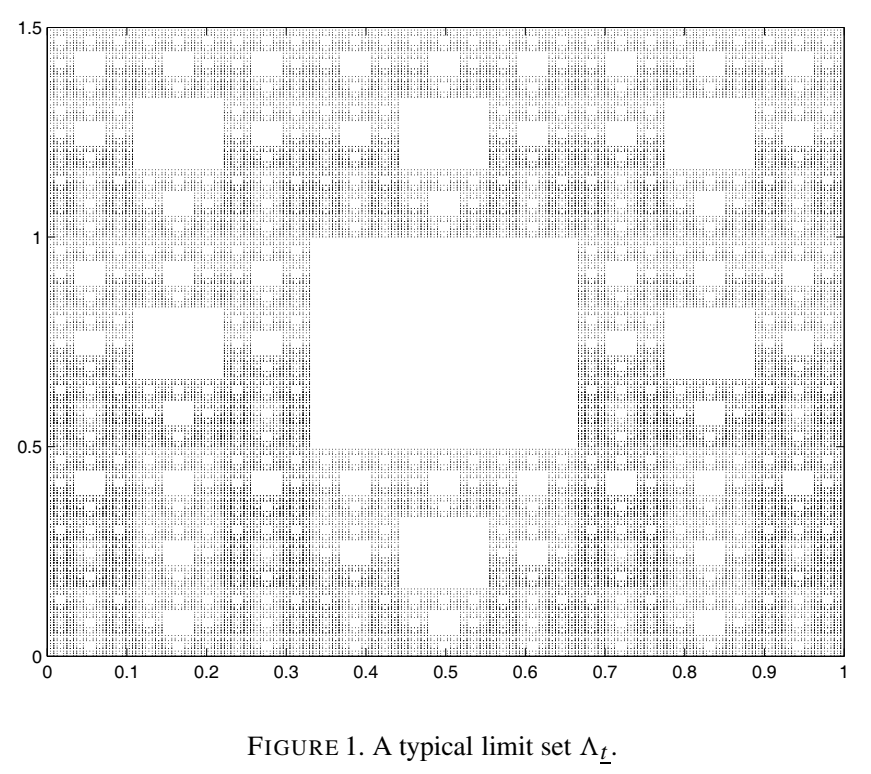

Let $\Sigma_{10}=\{0,1, \ldots, 9\}^{\mathbb{Z}^{+}}$denote the full shift on 10 symbols and let $\Pi_{\underline{t}}: \Sigma_{10} \rightarrow \Lambda_{\underline{t}}$ be the usual projection map. Let $\mu=\left(\frac{1}{12}, \frac{1}{12}, \frac{1}{12}, \frac{1}{12}, \frac{1}{6}, \frac{1}{6}, \frac{1}{12}, \frac{1}{12}, \frac{1}{12}, \frac{1}{12}\right)^{\mathbb{Z}^{+}}$be a Bernoulli measure on $\Sigma_{10}$. To show that $\Lambda_{\underline{t}}$ has non-zero Lebesgue measure it suffices to show that $v:=\Pi_{\underline{t}} \mu$ is absolutely continuous. By construction, $v$ projects to Lebesgue measure on the unit interval in the $x$-axis; thus it suffices to show the conditional measure $v_{t}, x$ on Lebesgue almost every vertical line $\{x\} \times \mathbb{R}$ is absolutely continuous.

Let $\Sigma_{3}=\{0,1,2\}^{\mathbb{Z}^{+}}$be the full shift on 3 symbols coding the horizontal coordinate. In particular, there is a natural semi-conjugacy $p: \Sigma_{10} \rightarrow \Sigma_{3}$ given by $p(\omega)_{n}=i\left(\omega_{n}\right)$ where $\left.i\right|_{\{0,1,2,3\}} \equiv 0 ;\left.i\right|_{\{4,5\}} \equiv 1$; and $\left.i\right|_{\{6,7,8,9\}} \equiv 2$. Then $p \mu=\bar{\mu}=\left(\frac{1}{3}, \frac{1}{3}, \frac{1}{3}\right)^{\mathbb{Z}^{+}}$ is the Bernoulli measure on $\Sigma_{3}$. Given $\xi \in \Sigma_{3}$ let $\mu_{\xi}$ denote the induced measure on $p^{-1}(\xi)$. Clearly, if $\Pi_{\underline{t}, \xi}: p^{-1}(\xi) \rightarrow\{x\} \times \mathbb{R}$ is the restriction of $\Pi_{\underline{t}}$, then by construction $\Pi_{t, \xi} \mu_{\xi}=v_{t}, x$. We also let $\pi: \Sigma_{3} \rightarrow[0,1]$ be the natural projection from $\Sigma_{3}$ to the $x$-axis given by $\pi(\xi)=\sum_{n=0}^{\infty} \xi_{n}\left(\frac{1}{3}\right)^{n+1}$. The analogue of transversality is that there exists $C>0$ such that

$$
\Delta_{\xi}(r ; \omega, \tau):=\operatorname{leb}\left\{\underline{t} \in[0,1]^{2}:\left|\Pi_{\underline{t}, \xi}(\omega)-\Pi_{\underline{t}, \xi}(\tau)\right| \leq r\right\} \leq C 3^{|\omega \wedge \tau|} r, \quad \text { for } r>0 .
$$

This can be seen by a simple adjustment of the arguments in [3, Lemma 3.1] and [4, Proposition 3.1 Part (i)]. However, in our special case it can be easily verified directly, as follows.

Let $\omega, \tau \in p^{-1}(\xi)$ with $|\omega \wedge \tau|=n$; that is, $\tau_{i}=\omega_{i}$ for $i<n$ and $\tau_{n} \neq \omega_{n}$. Since $\omega, \tau \in p^{-1}(\xi)$ we have $i\left(\omega_{n}\right)=i\left(\tau_{n}\right)$ for all $n$, and $\Pi_{t, \xi}(\omega)-\Pi_{t, \xi}(\tau)=$ $\left(0, \phi_{t, \xi}(\omega, \tau)\right)$, where

$$
\phi_{\underline{t}, \xi}(\omega, \tau)=3^{-n}\left(\left(t_{j\left(\omega_{n}\right)}-t_{j\left(\tau_{n}\right)}\right)+\sum_{k=1}^{\infty} 3^{-k}\left(t_{j\left(\omega_{k+n}\right)}-t_{j\left(\tau_{k+n}\right)}\right)\right) .
$$


Here we let $\left.j\right|_{\{0,4,6\}} \equiv 0,\left.j\right|_{\{1,7\}} \equiv 1,\left.j\right|_{\{2,8\}} \equiv 2,\left.j\right|_{\{3,5,9\}} \equiv 3$, and $t_{0}=0, t_{3}=1$ for convenience. If $\left\{j\left(\omega_{n}\right), j\left(\tau_{n}\right)\right\}=\{0,3\}$, then

$$
\left|\phi_{\underline{t}, \xi}(\omega, \tau)\right| \geq 3^{-n}\left(1-\sum_{k=1}^{\infty} 3^{-k}\right)=3^{-n} / 2
$$

in view of $t_{j} \in[0,1]$ for all $j$, and (1) follows. Otherwise, let $j \in\left\{j\left(\omega_{n}\right), j\left(\tau_{n}\right)\right\} \cap\{1,2\}$. Then

$$
\left|\frac{\partial \phi_{\underline{t}, \xi}(\omega, \tau)}{\partial t_{j}}\right| \geq 3^{-n}\left(1-\sum_{k=1}^{\infty} 3^{-k}\right)=3^{-n} / 2,
$$

which also implies (1).

Now we use (1) to prove that $v_{\underline{t}, x}$ is absolutely continuous for almost every $x$. For a sequence $\xi \in \Sigma_{3}$ we define $n_{i}(\xi)$ to be the number of $i$ 's in the first $n$ terms of $\xi$. By the strong law of large numbers, given $\epsilon, \delta>0$ we can use Egorov's theorem to choose a set $X \subset[0,1]$ of measure leb $(X)>1-\epsilon$ (equivalently $\left.\bar{\mu}\left(\pi^{-1} X\right)>1-\epsilon\right)$ such that there exists $N \in \mathbb{N}$ where for $n \geq N, n_{i}(\xi) \geq\left(\frac{1}{3}-\delta\right) n$, for $i=0,1,2$. We can bound

$$
\begin{aligned}
& \int_{[0,1]^{2}} \int_{X}\left(\int_{\{x\} \times \mathbb{R}} \underline{D}\left(v_{\underline{t}, x}\right)(y) d v_{\underline{t}, x}(y)\right) d(\mathrm{leb})(x) d \underline{\underline{t}} \\
& \quad \leq \liminf _{r \rightarrow 0} \frac{1}{2 r} \int_{\pi^{-1} X}\left(\int_{p^{-1}(\xi)} \int_{p^{-1}(\xi)} \Delta_{\xi}(r ; \omega, \tau) d \mu_{\xi}(\omega) d \mu_{\xi}(\tau)\right) d \bar{\mu}(\xi) \\
& \quad \leq C \int_{\pi^{-1} X}\left(\sum_{n=0}^{\infty} \sum_{\tau_{0}, \ldots, \tau_{n-1}} \mu_{\xi}\left[\tau_{0}, \ldots, \tau_{n-1}\right]^{2} 3^{n}\right) d \bar{\mu}(\xi) \\
& \quad \leq C \int_{\pi^{-1} X}\left(\sum_{n=0}^{\infty} 4^{-n_{0}(\xi)} 2^{-n_{1}(\xi)} 4^{-n_{2}(\xi)} 3^{n}\right) d \bar{\mu}(\xi) \\
& \quad \leq C C_{1}+C \sum_{n=N}^{\infty}\left(4^{-(2 / 3-2 \delta)} 2^{-(1 / 3-\delta)} 3\right)^{n}
\end{aligned}
$$

for some $C_{1}>0$ bounding the first $N$ terms of the series, and observe that the series is finite for $\delta$ sufficiently small. This implies the desired (typical) absolute continuity, as in the main article.

We have proved the following.

THEOREM A. For almost every $\underline{\underline{t}} \in[0,1]^{2}$ the limit set $\Lambda_{\underline{t}}$ has positive Lebesgue measure and empty interior.

Remark. We can also construct examples with fewer similarities using different contraction rates. Let $0<\lambda<\frac{1}{2}$ and $\underline{t}=\left(t_{1}, t_{2}, t_{3}\right) \in[0,1]^{3}$. Consider the six similarities of $\mathbb{R}^{2}$ defined by

$$
\begin{array}{ll}
T_{0}(x, y)=(\lambda x, \lambda y), & T_{3}(x, y)=(\lambda+\lambda x, \lambda y) \\
T_{1}(x, y)=\left(\lambda x, \lambda y+t_{1}\right), & T_{4}(x, y)=\left(\lambda+\lambda x, \lambda y+t_{1}\right) \\
T_{2}(x, y)=\left(\lambda x, \lambda y+t_{2}\right), & T_{5}(x, y)=\left(2 \lambda+(1-2 \lambda) x,(1-2 \lambda) y+t_{3}\right) .
\end{array}
$$


Let $\Lambda_{\underline{t}}$ again denote the self-similar set. Let $\mu=\left(\frac{\lambda}{3}, \frac{\lambda}{3}, \frac{\lambda}{3}, \frac{\lambda}{2}, \frac{\lambda}{2},(1-2 \lambda)\right)^{\mathbb{Z}^{+}}$be the Bernoulli measure on $\Sigma_{6}$. Let $\bar{\mu}=(\lambda, \lambda,(1-2 \lambda))^{\mathbb{Z}^{+}}$denote the induced measure on $\Sigma_{3}$. The proof of Theorem A can be adapted to this setting provided

$$
-(h(\mu)-h(\bar{\mu}))=-\lambda \log 2-\lambda \log 3 \leq 2 \lambda \log \lambda+(1-2 \lambda) \log (1-2 \lambda),
$$

which is true provided $\lambda$ is sufficiently close to $\frac{1}{2}$. More precisely, we have the following result.

THEOREM B. If $\lambda \in\left(0.4759, \frac{1}{2}\right)$ then for almost every $\underline{t} \in[0,1]^{3}$ the limit set $\Lambda_{\underline{t}}$ has positive Lebesgue measure and empty interior.

\section{REFERENCES}

[1] T. Jordan and M. Pollicott. Properties of measures supported on fat Sierpinski carpets, this issue.

[2] Y. Peres and B. Solomyak. Problems on self-similar and self-affine sets; an update. Progr. Prob. 46 (2000), 95-106.

[3] K. Falconer. The Hausdorff dimension of self-affine fractals. Math. Proc. Cambridge Philos. Soc. 103 (1988), 339-350.

[4] B. Solomyak. Measure and dimension for some fractal families. Math. Proc. Cambridge Philos. Soc. 124 (1998), 531-546. 